\title{
Prognostic value of the creatinine-albumin ratio in acute pancreatitis debridement
}

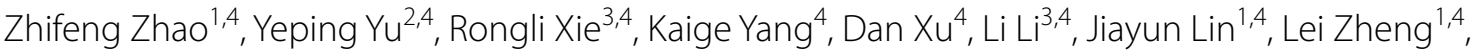

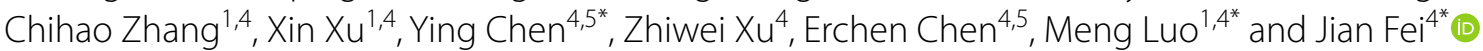

\begin{abstract}
Background: Increases in the levels of serum C-reactive protein (CRP) and creatinine ( $\mathrm{Cr}$ ) and decreases in those of albumin (Alb) are commonly observed in acute pancreatitis (AP). We aimed to evaluate the efficacy of the Cr/Alb and CRP/Alb ratios in the prediction of surgical treatment effect in AP patients.
\end{abstract}

Methods: This study retrospectively analyzed clinical data obtained from 140 AP patients who underwent debridement from January 2008 to November 2018 in Shanghai Ruijin Hospital. The Cr/Alb and CRP/Alb ratios at admission and before surgery were assessed in the analysis of clinical statistics, prediction of prognoses, and logistic regression analysis.

Results: The admission Cr/Alb had the best predictive value of the four ratios. This value was significantly higher in patients with re-operation and those who died $(P<0.05)$ and was correlated with the Acute Physiology and Chronic Health Evaluation (APACHE II) score, admission CRP/Alb, preoperative Cr/Alb, and post-operative complications. The admission Cr/Alb could predict the risk of AP-related re-operation and mortality with sensitivities, specificities and areas under the curve of $86.3 \%, 61.7 \%$ and 0.824 , and $73.4 \%, 81.3 \%$ and 0.794 , respectively. At a cut-off value of 3.43 , admission $\mathrm{Cr} / \mathrm{Alb}$ values were indicative of a worse clinical state, including impaired laboratory test values, APACHE II scores, rates of post-operative complications and re-operation, and mortality $(P<0.05)$. In the logistic regression analysis, admission $\mathrm{Cr} / \mathrm{Alb}$ values were independently related to the APACHE II score, post-operative renal failure, and mortality.

Conclusion: Cr/Alb is a novel but promising, easy-to-measure, reproducible, non-invasive prognostic score for the prediction of the effect of debridement in AP patients.

Keywords: Acute pancreatitis, C-reactive protein, Creatinine, Albumin

*Correspondence: bichatlion@163.com; luosh9hospital@sina.com; fj10777@rjh.com.cn

${ }^{1}$ Department of General Surgery, Shanghai Ninth People's Hospital Affiliated to Shanghai Jiao Tong University School of Medicine, No.639, Zhizaoju Road, Huangpu District, Shanghai, People's Republic of China

${ }^{4}$ Pancreatic Treatment Center, Ruijin Hospital Affiliated to Shanghai Jiao Tong University School of Medicine, No. 197, Ruijin No.2 Road, Huangpu District, Shanghai, People's Republic of China

${ }^{5}$ Department of Emergency, Ruijin Hospital Affiliated to Shanghai Jiao Tong University School of Medicine, No. 197, Ruijin No.2 Road, Huangpu District, Shanghai, People's Republic of China

Full list of author information is available at the end of the article

\section{Background}

Acute pancreatitis (AP) is a severe abdominal disorder characterized by sudden onset, rapid progression, and high mortality [1]. During AP treatment, debridement is often required among those with severe infection, peripancreatic necrosis, ineffective conservative treatment, and the use of minimally invasive approaches such as percutaneous catheter drainage [2]. However, due to the wide range of inflammation and surgical debridement and potential for severe infection, the surgical treatment of such patients is associated with a complication rate of 
$34-95 \%$, re-operation rate of $60-70 \%$, and perioperative mortality values of $11-39 \%[3,4]$. Considering the high risk of morbidity, mortality and poor prognoses during AP-related debridement, it is of great significance to evaluate and predict the risk of surgery before it is performed.

At present, several scores are used in the assessment of AP severity, such as Ranson's Criteria and the Acute Physiology and Chronic Health Evaluation II Score (APACHE II). However, the calculation of those scores requires the use of numerous parameters and very complicated algorithms, due to which their use is restricted in clinical practice $[5,6]$. Therefore, there is an urgent demand for a more simple, rapid and real-time tool for the prediction of disease severity and debridement efficiency.

Previous studies have shown that serum C-reactive protein $(\mathrm{CRP})$, creatinine $(\mathrm{Cr})$, and albumin $(\mathrm{Alb})$ are related to AP severity and prognoses [7-12]. While their predictive value is unsatisfactory when used alone, their use, in combination, can effectively improve the sensitivity of prediction [13]. As the levels of CRP and $\mathrm{Cr}$ are often elevated, and those of Alb are often decreased in AP, a ratio including CRP or $\mathrm{Cr}$ and Alb could magnify the sensitivity of examination. CRP/Alb has previously been investigated in AP patients. At a cut-off value of 16.28 , the mortality value in the higher CRP/Alb groups was 19.3 times that in the lower CRP/Alb group [14], suggesting its predictive value. $\mathrm{Cr}$ is also a valuable AP marker. However, no studies till date have investigated a ratio including $\mathrm{Cr}$ and Alb. Accordingly, this study aimed to explore the predictive value of $\mathrm{Cr} / \mathrm{Alb}$, and compare it with that of CRP/Alb in the assessment of surgical treatment in AP patients.

\section{Method}

\section{Patients}

A retrospective analysis was performed among AP patients who underwent surgery in Shanghai Ruijin Hospital from January 2008 to November 2019. Totally, 140 patients were enrolled in the study. All the procedures were implemented based on the principles of the Declaration of Helsinki, and the design of the work was reviewed and approved by the Ethics Committee of our hospital. As this is a retrospective research, the need for patient consent was waived by our institutional ethic committee.

The inclusion criteria were: (1) enrollment based on the Atlanta Classification criteria for AP that requires the presence of at least two of the following factors: (1) abdominal pain highly suggestive of AP, (2) elevations in the levels of serum amylase and/or lipase to more than three times the upper limit of normal, and (3) presence of radiological findings (ultrasonography or computerized tomography) characteristic of AP $[15,16]$; (2) performance of at least one debridement procedure for AP at our hospital. The exclusion criteria were: (1) presence of benign and malignant pancreatic tumors; (2) incomplete medical records; (3) age < 18 years or pregnant; (4) combination with other digestive system diseases.

All AP patients were diagnosed based on the presence of at least two of the following three criteria: (i) abdominal pain suggestive of AP, (ii) elevation in serum amylase and/or lipase $>3$ times the upper limit of normal, and (iii) computed tomography (CT) findings characteristic of AP. Presence of organ failure was assessed at admission and every $24 \mathrm{~h}$ during the hospitalization $[15,16]$. After admission, the patients received standard treatment, including gastrointestinal decompression, anti-infection, pain control, inhibition of pancreatic secretion, and fluid resuscitation. Antibiotics were used empirically first, and then adjusted using the results of microbial body fluid cultivation. Surgical therapy followed the step-up principle, comprising anti-infection therapy, minimally invasive approaches, and laparotomy. All surgery-related decision-making was made through the discussion of a multiple disciplinary team comprising specialists from the Departments of General Surgery, Critical Care Medicine, Imaging, and Anesthesiology. All debridement procedures were performed by experienced surgeons from a specialized pancreatitis team.

\section{Data collection}

Data were collected from the history system in our hospital. The laboratory examination values of CRP, $\mathrm{Cr}$ and Alb were collected separately at admission and within $24 \mathrm{~h}$ before the first debridement. Other variables included demographics, etiology, interventions, surgical complications, and outcomes.

\section{Definition}

The presence of infected pancreatic necrosis was defined as purulent culture. Hemorrhage was diagnosed by a reduction $>2 \mathrm{~g} / \mathrm{dL}$ and/or significant hemodynamic deterioration [17]. Shock was defined as a requirement for positive inotropic drug use or a systolic blood pressure $<90 \mathrm{mmHg}$. Respiratory failure was diagnosed as the ratio of arterial oxygen pressure and inspired oxygen concentration $\left(\mathrm{PaO}_{2} / \mathrm{FiO}_{2}\right) \leq 300 \mathrm{mmHg}$. Renal failure was defined as a serum $\mathrm{Cr}$ level $>176 \mu \mathrm{mol} / \mathrm{L}(2 \mathrm{mg} / \mathrm{dL})$. Other complications were defined in accordance with the Atlanta Classification of AP [18].

\section{Statistical analysis}

All statistical analyses were performed using SPSS 25.0. The Shapiro-Wilk test was used to evaluate the 
normal distribution of the data. Categorical variables were expressed as numbers and percentages, and examined by $x^{2}$ test and Pearson's test. Numerical data were expressed as $\bar{x} \pm s$, and the presence of significant differences was examined by Student's $t$ test. Receiver operating characteristic (ROC) curves and areas under the curve (AUCs) were used to assess the predictive value for prognoses. Logistic regression was performed in the multivariate analysis for the evaluation of independent risk factors. In the statistical analysis, $P<0.05$ was considered statistically significant.

\section{Results}

\section{General clinical characteristics}

After the exclusion of 31 patients, the study enrolled 140 patients (Fig. 1), including 98 men (70.00\%) and 42 women $(30.00 \%)$, with an average age of $49.88 \pm 13.94$ years. In terms of etiology, there were seven post-endoscopic retrograde cholangio-pancreatography cases, 66 gallstone cases, 28 hyperlipidemia cases, and 39 idiopathic AP cases. Of the 140 AP patients, 60 (42.86\%) underwent re-operation, including 40 cases of debridement, 13 of hemostasis, and seven of debridement combined with hemostasis. Complications occurred after debridement in 90 patients (64.29\%), including sepsis in 37 patients, electrolyte disorders in 31 patients, respiratory failure in 40 patients, hemorrhage in 34 patients, shock in 36 patients and renal failure in 34 patients. Finally, 16 patients died during the perioperative period (11.43\%), as shown in Table 1.

\section{Distribution of the $\mathrm{Cr} / \mathrm{Alb}$ and CRP/Alb ratios in groups of re-operation and mortality}

The admission CRP/Alb was only significantly higher in the re-operation group $(P<0.05)$. The distributions of the preoperative CRP/Alb values showed no significant differences between both subgroups $(P>0.05)$. The admission $\mathrm{Cr} / \mathrm{Alb}$ was significantly higher in the re-operation group $(P<0.001)$ and mortality group $(P=0.011)$. The preoperative $\mathrm{Cr} / \mathrm{Alb}$ was significantly higher only in the mortality group $(P<0.01)$, as shown in Table 2 . The APACHE II score was significantly higher both in the reoperation and mortality groups, with $P<0.05$, similar to the admission $\mathrm{Cr} / \mathrm{Alb}$. As a result, admission $\mathrm{Cr} / \mathrm{Alb}$ and $\mathrm{CRP} / \mathrm{Alb}$ and preoperative $\mathrm{Cr} / \mathrm{Alb}$ were chosen for further analysis.

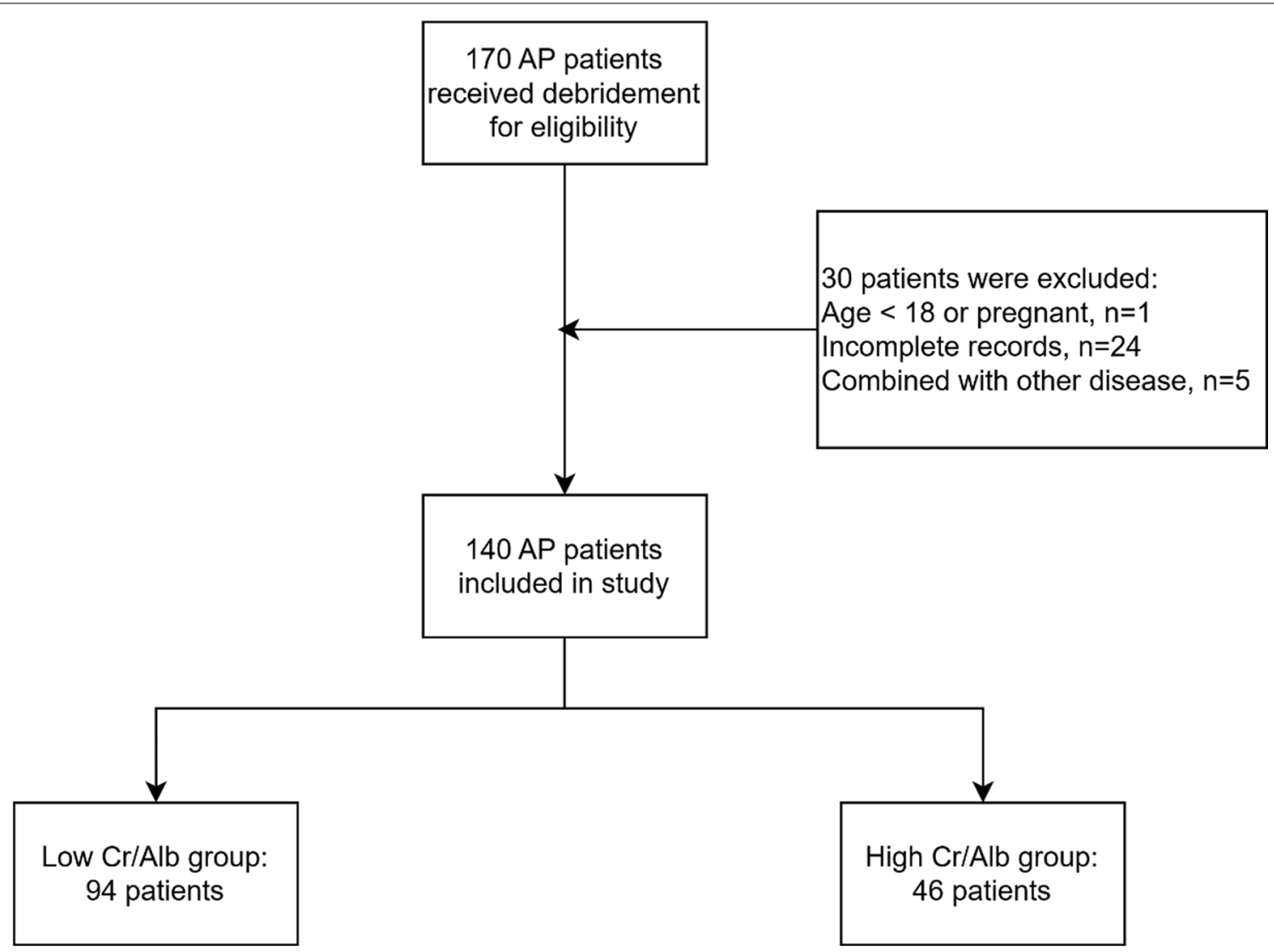

Fig. 1 Flow chart of the research 
Table 1 Demographic and clinical characteristics of AP patients

\begin{tabular}{ll}
\hline Variables & Patients ( $\mathbf{n = 1 4 0 )}$ \\
\hline Age, years & $49.88 \pm 13.94$ \\
Male/female, no. (\%) & $98(70.00) / 42(30.00)$ \\
Etiology, no. (\%) & \\
Post-ERCP & $7(5.00)$ \\
Gallstone & $66(47.14)$ \\
Hypertriglyceridemia & $28(20.00)$ \\
Idiopathic & $39(27.86)$ \\
Re-operation, no. (\%) & $60(42.86)$ \\
Debridement & $40(28.57)$ \\
Hemostasis & $13(9.29)$ \\
Debridement \& hemostasis & $7(5.00)$ \\
Post-operative complications no. (\%) & $90(64.29)$ \\
Sepsis & $37(26.43)$ \\
Electrolyte disorder & $31(22.14)$ \\
Raspatory failure & $40(28.57)$ \\
Hemorrhage & $34(24.29)$ \\
Shock & $36(25.71)$ \\
Renal failure & $34(24.29)$ \\
Outcomes, no. (\%) & \\
Survival & $124(88.57)$ \\
Death & $16(11.43)$ \\
\hline
\end{tabular}

Correlation analysis between the ratios and general clinical statistics of the AP patients

Table 3 shows the correlation between the three ratios and the patients' general clinical characteristics. The admission CRP/Alb was correlated with admission $\mathrm{Cr}(\mathrm{r}=0.243, P=0.013)$, admission CRP $(\mathrm{r}=0.959$, $P<0.001)$, admission Alb $(\mathrm{r}=0.284, P=0.022)$, preoperative CRP $(r=0.295, P=0.017)$, admission $\mathrm{Cr} / \mathrm{Alb}$ $(\mathrm{r}=0.241, P=0.014)$, and intensive care unit (ICU) duration $(\mathrm{r}=0.294, P=0.003)$. The admission $\mathrm{Cr} / \mathrm{Alb}$ was correlated with the APACHE II score $(r=0.495$, $P<0.001)$, admission blood urea nitrogen $(\mathrm{r}=0.815$, $P<0.001)$, admission Alb $(\mathrm{r}=-0.221, P=0.009)$, admission $\mathrm{Cr}(\mathrm{r}=0.983, P<0.001)$, preoperative $\mathrm{Cr}$ $(\mathrm{r}=0.375, P=0.002)$, admission CRP/Alb $(\mathrm{r}=0.241$, $P=0.014)$, preoperative $\mathrm{Cr} / \mathrm{Alb}(\mathrm{r}=0.428, P<0.001)$, and post-operative complications $(r=0.382, P=0.002)$. The preoperative $\mathrm{Cr} / \mathrm{Alb}$ was correlated with admission blood urea nitrogen $(\mathrm{r}=0.326, P=0.010)$, admission $\mathrm{Cr}(\mathrm{r}=0.412, P=0.001)$, admission $\mathrm{Cr} / \mathrm{Alb}(\mathrm{r}=0.428$, $P<0.001)$, and ICU duration $(\mathrm{r}=-0.254, P=0.040)$. Of those ratios, admission $\mathrm{Cr} / \mathrm{Alb}$ presented the greatest application value through its correlation with most of the eight variables, including the other two ratios, simultaneously.

\section{Predictive value of $\mathrm{Cr} / \mathrm{Alb}$ for re-operation and mortality} Figures 2 and 3 show the ROC curve of the admission $\mathrm{Cr} / \mathrm{Alb}$ in the prediction of re-operation and mortality. According to the ROC curve, we calculated the predictive values including cut-off values, sensitivities, specificities, and AUCs (Table 4).

The admission CRP/Alb showed the lowest efficacy, with sensitivity, specificity and AUC values of $76.1 \%$, $58.3 \%$ and 0.691 for re-operation, and $49.5 \%, 75.0 \%$ and 0.635 for mortality, respectively. The admission $\mathrm{Cr} / \mathrm{Alb}$ had a better predictive value, with a sensitivity of $86.3 \%$, specificity of $61.7 \%$, and AUC of 0.724 for re-operation, and a sensitivity of $73.4 \%$, specificity of $81.3 \%$ and AUC of 0.794 for mortality. The predictive value of the preoperative $\mathrm{Cr} / \mathrm{Alb}$ for mortality was more meaningful, with a sensitivity of $100.0 \%$, specificity of $72.1 \%$ and AUC of 0.872 , with a cut-off of 2.57. Additionally, its predictive value for re-operation was also satisfactory, with sensitivity, specificity and AUC values of $55.6 \%$, $68.4 \%$ and 0.606 respectively. In comparison, the sensitivity, specificity and AUC values of the APACHE II score were $82.6 \%, 58.3$ and 0.716 for re-operation, and $50.9 \%, 92.3 \%$ and 0.713 for mortality, respectively. In general, the predictive values of the $\mathrm{Cr} / \mathrm{Alb}$ ratio and APACHE II score were basically the same.

Table 2 Distribution of ratio variables in groups of re-operation and perioperative death

\begin{tabular}{|c|c|c|c|c|c|c|}
\hline \multirow[t]{2}{*}{ Variables } & \multicolumn{3}{|c|}{ Re-operation group } & \multicolumn{3}{|c|}{ Mortality group } \\
\hline & Single-operation & Re-operation & $P$ value & Survival & Death & $P$ value \\
\hline Admission CRP/Alb & $2.90 \pm 3.02$ & $4.63 \pm 2.80$ & $0.005^{*}$ & $3.31 \pm 2.88$ & $4.98 \pm 3.96$ & 0.074 \\
\hline Admission Cr/Alb & $3.07 \pm 3.21$ & $7.31 \pm 7.57$ & $<0.001^{* *}$ & $4.22 \pm 5.21$ & $10.11 \pm 8.09$ & $0.011^{*}$ \\
\hline Preoperative CRP/Alb & $2.82 \pm 2.92$ & $2.50 \pm 2.30$ & 0.636 & $2.72 \pm 2.86$ & $2.53 \pm 1.46$ & 0.748 \\
\hline Preoperative $\mathrm{Cr} / \mathrm{Alb}$ & $3.83 \pm 4.79$ & $6.45 \pm 8.27$ & 0.147 & $3.59 \pm 4.62$ & $11.47 \pm 10.17$ & $<0.01^{* *}$ \\
\hline APACHE II & $12.57 \pm 6.55$ & $18.80 \pm 8.65$ & $<0.001^{* *}$ & $14.89 \pm 8.11$ & $20.62 \pm 7.24$ & $0.016^{*}$ \\
\hline
\end{tabular}

*Means $P<0.05,{ }^{* *}$ means $P<0.01$ 
Table 3 Correlation analysis between ratios and general clinical statistics of AP patients

\begin{tabular}{|c|c|c|c|c|c|c|}
\hline \multirow[t]{2}{*}{ Variables } & \multicolumn{2}{|c|}{ Admission CRP/Alb } & \multicolumn{2}{|c|}{ Admission Cr/Alb } & \multicolumn{2}{|c|}{ Preoperative $\mathrm{Cr} / \mathrm{Alb}$} \\
\hline & $r$ & $P$ & $r$ & $P$ & $r$ & $P$ \\
\hline Age & -0.143 & 0.149 & -0.107 & 0.209 & 0.137 & 0.276 \\
\hline Gender & -0.044 & 0.729 & 0.068 & 0.590 & -0.009 & 0.943 \\
\hline BMI & 0.230 & 0.051 & 0.073 & 0.495 & -0.067 & 0.645 \\
\hline APACHE ॥ & 0.176 & 0.093 & 0.495 & $0.000^{* *}$ & 0.325 & 0.012 \\
\hline Admission WBC & 0.142 & 0.171 & 0.174 & 0.051 & -0.061 & 0.648 \\
\hline Admission $\mathrm{Hb}$ & 0.144 & 0.153 & -0.065 & 0.450 & -0.044 & 0.736 \\
\hline Admission TB & -0.004 & 0.966 & 0.151 & 0.078 & 0.116 & 0.375 \\
\hline Admission BUN & 0.087 & 0.391 & 0.815 & $0.000^{* *}$ & 0.326 & $0.010^{*}$ \\
\hline Admission Alb & -0.111 & 0.264 & -0.221 & $0.009^{* *}$ & -0.186 & 0.140 \\
\hline Admission $\mathrm{Cr}$ & 0.243 & $0.013^{*}$ & 0.983 & $0.000^{* *}$ & 0.412 & $0.001^{* *}$ \\
\hline Admission CRP & 0.959 & $0.000^{* *}$ & 0.182 & 0.083 & -0.026 & 0.845 \\
\hline Preoperative Alb & 0.284 & $0.022^{*}$ & -.046 & 0.716 & -0.121 & 0.337 \\
\hline Preoperative $\mathrm{Cr}$ & 0.048 & 0.704 & 0.375 & $0.002^{* *}$ & 0.984 & 0.000 \\
\hline Preoperative CRP & 0.295 & $0.017^{*}$ & -0.123 & 0.330 & -0.126 & 0.318 \\
\hline Admission CRP/Alb & / & / & 0.241 & $0.014^{*}$ & 0.005 & 0.971 \\
\hline Admission Cr/Alb & 0.241 & $0.014^{*}$ & / & / & 0.428 & $0.000^{* *}$ \\
\hline Preoperative Cr/Alb & 0.005 & 0.971 & 0.428 & $0.000^{* *}$ & / & / \\
\hline Post-operative complications & 0.132 & 0.295 & 0.382 & $0.002^{* *}$ & 0.230 & 0.065 \\
\hline ICU duration & 0.294 & $0.003^{* *}$ & 0.055 & 0.523 & -0.255 & $0.040^{*}$ \\
\hline
\end{tabular}

$B M I$ Body Mass Index, APACHE I/ Acute Physiology and Chronic Health Evaluation, WBC white blood cell, Hb hemoglobin, $T B$ total bilirubin, $B U N$ blood urea nitrogen, Alb Albumin, $C r$ creatinine, CRP C-reactive protein, ICU intensive care unit

*Means $P<0.05,{ }^{* *}$ means $P<0.01$

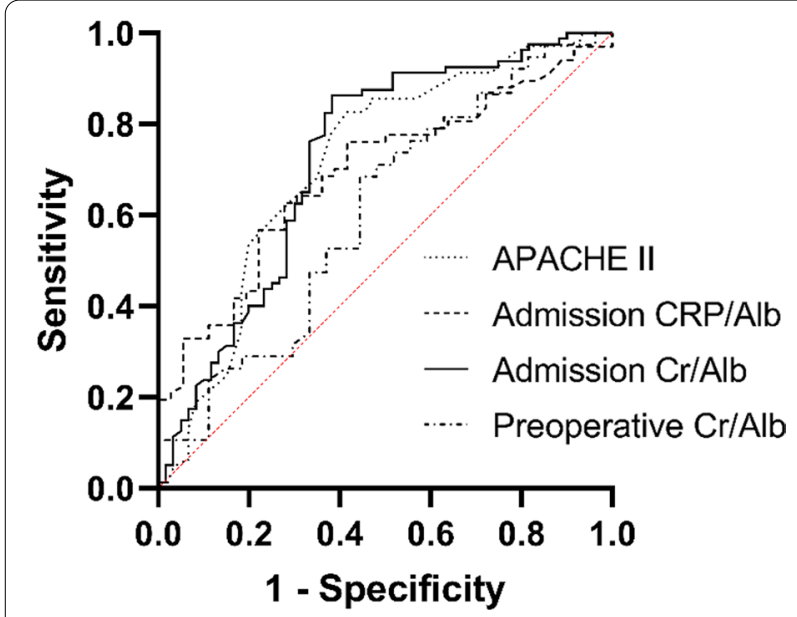

Fig. 2 The ROC curve of multiple variables in assessing the re-operation of AP debridement which including APACHE II, admission CRP/Alb, Admission Cr/Alb and Preoperative Cr/Alb. Among them the Admission Cr/Alb had the highest AUC (0.724), APACHE II the second highest (0.716), Admission CRP/Alb the third highest (0.691) and Preoperative Cr/Alb the lowest (0.606)

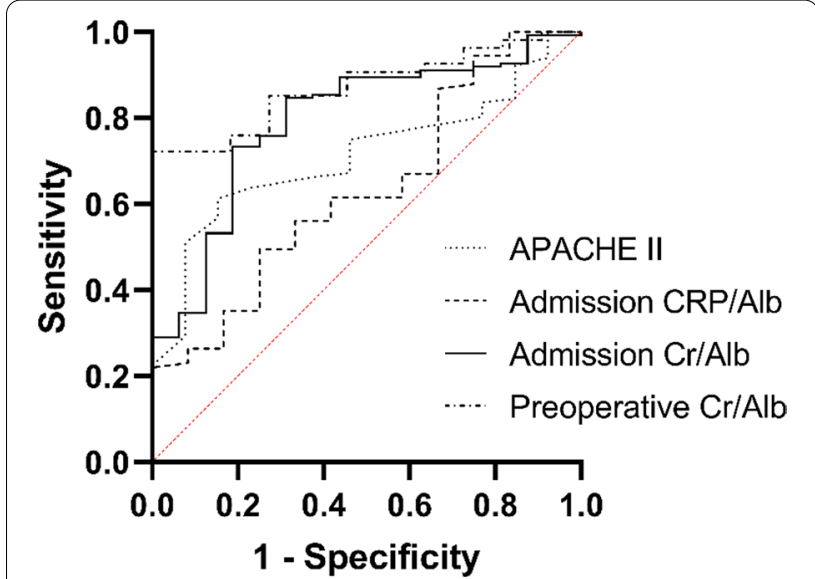

Fig. 3 The ROC curve of multiple variables in assessing the mortality of AP patients which including APACHE II, admission CRP/ Alb, Admission Cr/Alb and Preoperative Cr/Alb. Among them the Preoperative Cr/Alb had the highest AUC (0.872), Admission Cr/Alb the second highest (0.794), APACHE II the third highest (0.713) and Admission CRP/Alb the lowest (0.635) 
Table 4 Predictive values of ratio valuables for re-operation and mortality in AP patients

\begin{tabular}{|c|c|c|c|c|c|c|c|c|}
\hline \multirow[t]{2}{*}{ Ratio } & \multicolumn{4}{|c|}{ Re-operation } & \multicolumn{4}{|c|}{ Mortality } \\
\hline & AUC & Sensitivity (\%) & Specificity (\%) & Cut-off value & AUC & Sensitivity (\%) & Specificity (\%) & Cut-off value \\
\hline $\begin{array}{l}\text { Admission } \\
\text { CRP/Alb }\end{array}$ & 0.691 & 76.1 & 58.3 & 4.41 & 0.635 & 49.5 & 75.0 & 7.69 \\
\hline Admission Cr/Alb & 0.724 & 86.3 & 61.7 & 3.29 & 0.794 & 73.4 & 81.3 & 3.43 \\
\hline Preoperative $\mathrm{Cr} / \mathrm{Alb}$ & 0.606 & 55.6 & 68.4 & 2.50 & 0.872 & 100.0 & 72.1 & 2.57 \\
\hline APACHE $\|$ & 0.716 & 82.6 & 58.3 & 16.5 & 0.713 & 50.9 & 92.3 & 12.5 \\
\hline
\end{tabular}

\section{Relationship between admission $\mathrm{Cr} / \mathrm{Alb}$ and clinical characteristics}

As suggested above, the cut-off admission $\mathrm{Cr} / \mathrm{Alb}$ value was 3.29 for re-operation and 3.43 for mortality. The latter was chosen as the basis of grouping, as a ratio higher than 3.43 simultaneously satisfies two criteria. Of patients in the milder AP $(n=94)$ and severe AP $(n=46)$ group, those in the latter group with a $\mathrm{Cr} / \mathrm{Alb}$ value $\geq 3.43 \mathrm{had}$ a more intense heart rate and respiratory rate $(P<0.05)$, impaired laboratory data, including those pertaining to blood urea nitrogen, $\mathrm{Cr}$, Alb, white blood cells, platelets and the APACHE II score $(P<0.05)$, higher morbidity values associated with post-operative complications, including electrolyte disorders, respiratory failure, hemorrhage, shock and renal failure $(P<0.05)$, and higher rates of reoperation and mortality $(P<0.001)$. Besides, the interval times between onset and debridement were shorter in the severe group but still exceeded 3-4 weeks, in keeping with the Atlanta Classification criteria for AP (Table 5). These results suggest that a cut-off admission $\mathrm{Cr} / \mathrm{Alb}$ value of 3.43 has the potential to distinguish severe AP from milder AP.

\section{Logistic regression analysis of the admission $\mathrm{Cr} / \mathrm{Alb}$ in AP patients}

In the logistic regression analysis of the admission $\mathrm{Cr} / \mathrm{Alb}$ in AP patients, the APACHE II score, sepsis, electrolyte disorder, respiratory failure, hemorrhage, shock, renal failure, re-operation and mortality were enrolled after the elimination of interference variables through colinear diagnosis. The results suggested that the APACHE II score, renal failure and re-operation were independently related to the admission $\mathrm{Cr} / \mathrm{Alb}(P<0.05)$, as shown in Table 6. This indicated that the admission $\mathrm{Cr} / \mathrm{Alb}$ could predict the risk of re-operation and post-operative renal failure independently. AP patients with high admission $\mathrm{Cr} / \mathrm{Alb}$ values had 4.331 times the chance of renal failure and 3.824 times the chance of re-operation. Besides, the admission $\mathrm{Cr} / \mathrm{Alb}$ was correlated independently to the APACHE II score $(P=0.009)$, suggesting that the ratio could be a simpler substitute for the APACHE II score in clinical settings.

\section{Discussion}

In this study, we found that the $\mathrm{Cr} / \mathrm{Alb}$ ratio is a novel but promising, easy-to-measure, reproducible, non-invasive prognostic score that can be used for the prediction of the effect of debridement in AP patients.

AP is an emergency gastrointestinal condition, characterized by a rapid onset and poor prognoses, with mortality values of up to 40\% [19]. As AP often causes severe systemic inflammation and necrosis in the abdomen, debridement plays an important role in its treatment. However, the effect of surgery is not satisfactory owing to the complexity of pancreatic or peri-pancreatic infections, multiple organ attack, and violent systemic inflammation, due to which a large proportion of patients experience numerous post-operative complications, reoperation or even death. Therefore, it is important to evaluate patients' prognoses before surgery. Previous studies showed that CRP, Cr, and Alb had definite implications for systemic inflammatory response-related conditions such as AP $[20,21]$; however, their predictive value, when used alone, is not satisfactory. Therefore, some studies combined those parameters for an enhanced prediction effect $[14,22]$.

In our study, of all the admission and pre-operation $\mathrm{Cr} /$ $\mathrm{Alb}$ and CRP/Alb values, the admission Cr/Alb showed the best performance in several analyses, including the distribution analysis and correlation analysis, and had the best predictive value. An admission $\mathrm{Cr} / \mathrm{Alb}$ cut-off $\geq 3.43$ could predict worse prognoses in AP patients, including a more severe heart rate and respiratory rate, impaired levels of blood urea nitrogen, $\mathrm{Cr}$, Alb, white blood cells and platelets, APACHE II score, post-operative electrolyte disorders, respiratory failure, hemorrhage, shock, renal failure, re-operation rate, and mortality. In the logistic regression analysis, the admission $\mathrm{Cr} / \mathrm{Alb}$ was independently correlated with the APACHE II score, renal failure and re-operation. About the diagnosis value of $\mathrm{Cr} /$ $\mathrm{Alb}$, it is basically satisfying in predicting the outcomes of the AP debridement. In predicting the re-operation, the sensitivity, specificity, and AUC of the admission $\mathrm{Cr}$ / Alb reached $86.3 \%, 61.7 \%$, and 0.724 , respectively. And 
Table 5 Baseline characteristics of AP patients sorted by ratio of admission $\mathrm{Cr} / \mathrm{Alb}$

\begin{tabular}{|c|c|c|c|}
\hline Variables & $\begin{array}{l}\mathrm{Cr} / \mathrm{Alb}<3.43 \\
(\mathrm{n}=94)\end{array}$ & $\begin{array}{l}\mathrm{Cr} / \mathrm{Alb} \geq 3.43 \\
(n=46)\end{array}$ & $P$ value \\
\hline \multicolumn{4}{|c|}{ Demographic characteristics } \\
\hline Age, years & $49.73 \pm 13.00$ & $50.17 \pm 15.83$ & 0.862 \\
\hline Male, no. (\%) & $62(65.96)$ & $36(78.26)$ & 0.136 \\
\hline $\mathrm{BMI}, \mathrm{kg} / \mathrm{m}^{2}$ & $23.92 \pm 3.46$ & $24.83 \pm 3.95$ & 0.271 \\
\hline Etiology, no. (\%) & & & 0.317 \\
\hline Post-ERCP & $4(4.26)$ & $3(6.52)$ & \\
\hline Gallstone & $47(50.00)$ & $19(41.30)$ & \\
\hline Hypertriglyceridemia & $15(15.96)$ & $13(28.26)$ & \\
\hline Idiopathic & $28(29.79)$ & $11(23.91)$ & \\
\hline \multicolumn{4}{|l|}{ History, no. (\%) } \\
\hline Hypertension & $25(26.60)$ & $14(30.43)$ & 0.651 \\
\hline Alcohol & $22(23.40)$ & $12(26.09)$ & 0.744 \\
\hline Smoking & $21(22.34)$ & $11(23.91)$ & 0.770 \\
\hline Diabetes mellitus & $8(8.51)$ & $8(17.39)$ & 0.242 \\
\hline Fatty liver & $5(5.32)$ & $1(2.17)$ & 0.536 \\
\hline$C A D$ & $4(4.26)$ & $1(2.17)$ & 0.635 \\
\hline \multicolumn{4}{|l|}{ Clinical presentation } \\
\hline Interval time ${ }^{\mathrm{a}}$, days & $56.23 \pm 51.14$ & $35.18 \pm 37.13$ & $0.016^{*}$ \\
\hline Heart rate, bpm & $102.03 \pm 20.38$ & $116.45 \pm 23.10$ & $<0.001^{* *}$ \\
\hline Temperature, ${ }^{\circ} \mathrm{C}$ & $37.13 \pm 0.74$ & $37.27 \pm 1.02$ & 0.412 \\
\hline $\mathrm{MAP}, \mathrm{mmHg}$ & $61.18 \pm 37.54$ & $67.66 \pm 34.13$ & 0.319 \\
\hline Respiratory rate, bpm & $22.98 \pm 5.60$ & $25.95 \pm 7.55$ & $0.023^{*}$ \\
\hline \multicolumn{4}{|c|}{ Laboratory data in admission } \\
\hline Hemoglobin, g/L & $107.06 \pm 26.30$ & $107.3 \pm 32.06$ & 0.964 \\
\hline $\mathrm{BUN}, \mathrm{mmol} / \mathrm{L}$ & $5.64 \pm 3.13$ & $17.83 \pm 9.76$ & $<0.001^{* *}$ \\
\hline Creatinine, $\mu \mathrm{mol} / \mathrm{L}$ & $59.29 \pm 21.11$ & $277.50 \pm 187.02$ & $<0.001^{* *}$ \\
\hline $\mathrm{CRP}, \mathrm{mg} / \mathrm{L}$ & $102.52 \pm 73.29$ & $121.57 \pm 83.89$ & 0.262 \\
\hline Albumin, $\mathrm{g} / \mathrm{L}$ & $29.00 \pm 5.71$ & $26.80 \pm 5.30$ & $0.030^{*}$ \\
\hline WBC, $\times 10^{9}$ cells $/ \mathrm{L}$ & $11.94 \pm 5.39$ & $14.33 \pm 7.28$ & $0.039^{*}$ \\
\hline Platelet, $\times 10^{9}$ cells $/ \mathrm{L}$ & $239.08 \pm 114.78$ & $155.30 \pm 93.27$ & $<0.001^{* *}$ \\
\hline $\mathrm{TB}, \mu \mathrm{mol} / \mathrm{L}$ & $39.55 \pm 58.64$ & $51.12 \pm 67.60$ & 0.307 \\
\hline \multicolumn{4}{|l|}{ Score } \\
\hline APACHE ॥ & $12.59 \pm 6.76$ & $21.21 \pm 7.83$ & $<0.001^{* *}$ \\
\hline \multicolumn{4}{|c|}{ Post-operative complications, no. (\%) } \\
\hline Sepsis & $21(22.34)$ & $16(34.78)$ & 0.117 \\
\hline Electrolyte disorders & $14(14.89)$ & $17(36.96)$ & $0.003^{* *}$ \\
\hline Respiratory failure & $13(13.83)$ & $27(58.70)$ & $<0.001^{* *}$ \\
\hline Hemorrhage & $15(15.96)$ & $19(41.30)$ & $0.001^{* *}$ \\
\hline Shock & $12(12.77)$ & $24(52.17)$ & $<0.001^{* *}$ \\
\hline Renal failure & $7(7.45)$ & $27(58.70)$ & $<0.001^{* *}$ \\
\hline \multicolumn{4}{|l|}{ Outcome, no. (\%) } \\
\hline ICU duration, days & $58.75 \pm 75.13$ & $73.91 \pm 64.24$ & 0.251 \\
\hline Re-operation & $25(26.60)$ & $35(76.09)$ & $<0.001^{* *}$ \\
\hline Mortality & $3(3.19)$ & $13(28.26)$ & $<0.001^{* *}$ \\
\hline
\end{tabular}

CAD coronary artery disease, MAP mean arterial pressure, CRP C-reactive protein, TB total bilirubin, BUN blood urea nitrogen, APACHE I/ Acute Physiology and Chronic Health Evaluation II

*Means $P<0.05,{ }^{* *}$ means $P<0.001$

a Interval time means the interval days between onset of AP and first debridement 
Table 6 Logistic regression analysis of admission $\mathrm{Cr} / \mathrm{Alb}$ in AP patients

\begin{tabular}{llll}
\hline Variables & OR & $\mathbf{9 5 \%} \mathbf{C l}$ & $\boldsymbol{P}$ value \\
\hline APACHE II & 0.911 & $0.851-0.977$ & $0.009^{* *}$ \\
Sepsis & 0.806 & $0.248-2.617$ & 0.720 \\
Electrolyte disorder & 1.368 & $0.407-4.598$ & 0.613 \\
Respiratory failure & 0.996 & $0.268-3.702$ & 0.995 \\
Hemorrhage & 1.069 & $0.314-3.642$ & 0.915 \\
Shock & 1.350 & $0.369-4.963$ & 0.651 \\
Renal failure & 4.331 & $1.071-17.523$ & $0.040^{*}$ \\
Re-operation & 3.824 & $1.071-13.655$ & $0.039^{*}$ \\
Mortality & 2.548 & $0.381-17.021$ & 0.334 \\
\hline
\end{tabular}

*Means $P<0.05$, **means $P<0.01$

the variables of APACHE II were $82.6 \%, 58.3 \%$, and 0.716 , respectively. In the assessment of perioperative mortality, the sensitivity, specificity, and AUC of admission $\mathrm{Cr} /$ Alb were $73.4 \%, 81.3 \%$, and 0.794 , respectively. And those of APACHE II were $50.9 \%, 92.3 \%$ and 0.713 , respectively. Above all, our research suggests that the admission $\mathrm{Cr} /$ Alb has the potential to predict AP-related prognoses as a simpler alternative to the APACHE II score.

The development of AP is often accompanied by systemic inflammation, sepsis, and multiple organ failure. Therefore, the prognoses of AP are closely related to the severity of inflammation, nutrition intake, negative nitrogen balance, and functional reserve [23]. Of the various tests, CRP, $\mathrm{Cr}$ and Alb are the most relevant examinations. CRP is synthesized by the liver and responds to inflammation within a few hours [24]. Owing to its short half-life and high sensitivity, it is often used for the detection and assessment of inflammation [7, 25, 26]. Alb is a negative acute phase reactant synthesized by the liver, and its expression decreases during inflammatory reactions. In previous studies, Alb was shown to be related negatively to the severity of inflammation, disease prognoses, and mortality in AP [20,27]. Cr is an amino acid derivative produced by the metabolism of muscle tissues. It is filtered through the kidney and its levels are raised significantly in systemic inflammatory diseases [28]. An increase in the $\mathrm{Cr}$ level $48 \mathrm{~h}$ after admission is considered a marker of pancreatic necrosis, hypovolemia and renal insufficiency [11]. According to Wilkman et al. [29], increased Cr levels are independent risk factors for 90-day mortality in AP patients. Lipinski et al. [10] also reported that higher $\mathrm{Cr}$ values at admission and after $48 \mathrm{~h}$ were associated with a higher incidence of mortality.

In complicated disease like AP, a single biomarker usually could not provide accurate prognostic effect. As a result, more and more combinations of biomarkers were being applied recently. In AP, the most frequently used values were CRP/Alb, Neutrophil-lymphocyte ratio (NLR), Platelet-lymphocyte ratio (PLR), etc., which exceeded excellent diagnostic values in the assessment of AP. Suppiah et al. [30] discovered that the elevation of NLR at the first $48 \mathrm{~h}$ of admission was significantly associated with severe acute pancreatitis as a result of neutrophilia and lymphopenia during the systemic inflammatory response. The sensitivity and specificity of NLR to predict SAP achieved $63-90 \%$ and $50-57 \%$ respectively. PLR also showed good application value for mortality prediction with sensitivity of $73.3 \%$ and specificity of $99.22 \%$. Furtherly, it was found that the combination of PLR and NLR had the highest AUC in evaluating the prognosis of AP, with similar predictive value among other scoring systems [31]. Besides, Kaplan et al. [14] discovered that the CRP/Alb ratio could predict the mortality of AP patients with sensitivity of $92.1 \%$ and specificity of $58.0 \%$, while this ratio was also positively corelated with Ranson score, Atlanta classification etc. As mentioned above, those predictors reflected the severity of inflammatory status outstandingly. However, surgical intervention of AP means higher requirements for the surgical tolerance, higher risk of complications and mortality. Merely inflammatory factors might not be enough. Therefore, our study used $\mathrm{Cr}$ and Alb to focus mainly on the evaluation of surgical outcomes.

In our study, $\mathrm{Cr} / \mathrm{Alb}$ showed better performance than CRP/Alb. CRP level fluctuations are more intensive in $\mathrm{AP}$ and may lead to measurement-related difficulties and inaccuracies in severity evaluation. In comparison, $\mathrm{Cr}$ level trends are more stable, lending them more credibility for use as predictors. $\mathrm{Cr}$ also has the ability to reflect the pancreatic necrosis, protein decomposition metabolism and renal function. In pancreatic necrosis, a large amount of toxic substances and inflammatory factors would be released and directly attacking the kidney, leading to aggravated renal injury [12]. And the process in turn weakens the renal clearance ability and resulting in decreased surgical tolerance. Besides, Alb reflects a body's protein reserve more accurately. Several studies have given possible mechanistic explanations, (i) the inflammatory response produced by AP increases catabolism and tissue consumption; (ii) AP releases a large number of inflammatory factors such as interleukin-1, and interleukin- 6 leads to a decrease in albumin biosynthesis in liver; (iii) during the stress response, vascular permeability increases and albumin penetration into the tissue space [20,32]. In summary, $\mathrm{Cr}$ and Alb could assess the degree of inflammatory response, catabolism, and fundamental status. In AP patients with weaker status, serum albumin tends to decrease because of the impaired hepatic synthesis function, albumin exudation and increased consumption; and creatinine tends 
to increase because of the renal injury and pancreatic necrosis. The combination of the two markers could indicate the severity and prognosis of AP debridement more reliably and accurately.

This study is the first to compare admission and preoperative Cr/Alb and CRP/Alb values in the assessment of AP debridement. AP is a highly inflammatory and catabolic state that often leads to malnutrition. These features increase the risk of re-operation and mortality in debridement. Therefore, our study focused on surgical tolerance by examining the reserve capacity and nutritional status in contrast to previous studies that focused on severity of systemic inflammatory response. Patients with poor surgical tolerance usually face higher risk of re-operation and mortality, which requires more prudence in surgical decision-making. For surgeons, our study provides a simpler and more feasible tool for the evaluation of the basic state of AP patients before surgery-related decision-making. For ward managers, the $\mathrm{Cr} / \mathrm{Alb}$ value could aid in the identification of high-risk patients in advance and remind medical staff to strengthen the degree of ward care targeted at those patients. As the $\mathrm{Cr} / \mathrm{Alb}$ value could distinguish mild AP from its severe form, our results can be used by hospitals and health policymakers in the formulation of more efficient gradient treatment strategies for AP.

Our study has several limitations. First, its retrospective design may have led to a certain selection bias. Second, the study included only 140 cases enrolled from a single center, leading to insufficient reliability. Third, this study focused only on AP patients with debridement. In the future, we will include patients with nonsurgical treatment for further investigation.

In conclusion, as a clinical scoring system with high accuracy and simplicity, the admission $\mathrm{Cr} / \mathrm{Alb}$ value was superior in the assessment of the severity of AP with debridement. With the confirmation of our results in larger-scale investigations in the future, $\mathrm{Cr} / \mathrm{Alb}$ has the potential to improve the quality of the AP risk scoring system and prognostic prediction.

\section{Conclusion}

As a cheap, convenient and minimally invasive marker, $\mathrm{Cr} / \mathrm{Alb}$ is an independent predictor of re-operation and mortality for AP debridement. The Cr/Alb ratio has good predictive potential for AP debridement patients and its prognostic value is comparable with other diagnostic scores.

\section{Abbreviations}

CRP: C-reactive protein; Cr: Creatinine; Alb: Albumin; AP: Acute pancreatitis; APACHE II: Acute Physiology and Chronic Health Evaluation; CT: Computed tomography; ROC: Receiver operating characteristic; AUC: Areas under curve; ICU: Intensive care unit; NLR: Neutrophil-lymphocyte ratio; PLR: Plateletlymphocyte ratio.

\section{Acknowledgements}

We thank Wiley Editing Services for its linguistic assistance during the preparation of this manuscript.

\section{Authors' contributions}

ZZ was involved in the plan of program and drafted the manuscript. YY participated the data collection and analysis. RX, KY, DX and LL participated the data collection. $J L, L Z, C Z$ and $X X$ made substantial contributions to the manuscript and the analysis of data. YC, ZX, EC, ML and JF participated in drafting or revising the work. All authors have given final approval of the version to be published. All authors agreed to be accountable for all aspects of the manuscript. All authors read and approved the final manuscript.

\section{Funding}

This study is financially supported by the National Natural Science Fund of China (Project No. 81670581 to Jian FEI). The funding body had no role in the design of the study and collection, analysis, interpretation of data or preparation of the manuscript.

\section{Availability of data and materials}

The datasets generated and analysed during the current study are not publicly available as the data are being used in next study, but are available from the corresponding author on reasonable request.

\section{Ethics approval and consent to participate}

The experimental protocol was established, according to the ethical guidelines of the Helsinki Declaration and was approved by the Shanghai Jiao Tong University School of Medicine Ruijin Hospital Ethics Committee. Committee's reference number: 2017-119. Informed consent was waived for this non-interventional, observational, and retrospective study by the Ethics Committees, in which the patient data used were kept strictly confidential.

\section{Consent for publication}

Not applicable.

\section{Competing interests}

The authors declare that they have no competing interests.

\section{Author details}

${ }^{1}$ Department of General Surgery, Shanghai Ninth People's Hospital Affiliated to Shanghai Jiao Tong University School of Medicine, No.639, Zhizaoju Road, Huangpu District, Shanghai, People's Republic of China. ${ }^{2}$ School of Clinical Medicine, Ruijin Hospital Affiliated to Shanghai Jiao Tong University School of Medicine, Shanghai, People's Republic of China. ${ }^{3}$ Luwan Branch, Ruijin Hospital Affiliated to Shanghai Jiao Tong University School of Medicine, Shanghai, People's Republic of China. ${ }^{4}$ Pancreatic Treatment Center, Ruijin Hospital Affiliated to Shanghai Jiao Tong University School of Medicine, No. 197, Ruijin No.2 Road, Huangpu District, Shanghai, People's Republic of China. ${ }^{5}$ Department of Emergency, Ruijin Hospital Affiliated to Shanghai Jiao Tong University School of Medicine, No. 197, Ruijin No.2 Road, Huangpu District, Shanghai, People's Republic of China.

Received: 8 July 2020 Accepted: 29 November 2020

Published online: 09 December 2020

\section{References}

1. Lowenfels AB, Maisonneuve P, Sullivan T. The changing character of acute pancreatitis: epidemiology, etiology, and prognosis. Curr Gastroenterol Rep. 2009;11:97-103. https://doi.org/10.1007/s11894-009-0016-4.

2. Karakayali FY. Surgical and interventional management of complications caused by acute pancreatitis. World J Gastroenterol. 2014;20:13412-23. https://doi.org/10.3748/wjg.v20.i37.13412

3. Nieuwenhuijs VB, Besselink MGH, van Minnen LP, Gooszen HG. Surgical management of acute necrotizing pancreatitis: a 13-year experience 
and a systematic review. Scand J Gastroenterol Suppl. 2003. https://doi. org/10.1080/00855920310002799.

4. Rau B, Bothe A, Beger HG. Surgical treatment of necrotizing pancreatitis by necrosectomy and closed lavage: changing patient characteristics and outcome in a 19-year, single-center series. Surgery. 2005;138:28-39. https ://doi.org/10.1016/j.surg.2005.03.010.

5. Ranson JH. Etiological and prognostic factors in human acute pancreatitis: a review. Am J Gastroenterol. 1982;77:633-8.

6. Mortele KJ, Wiesner W, Intriere L, Shankar S, Zou KH, Kalantari BN, et al. A modified CT severity index for evaluating acute pancreatitis: improved correlation with patient outcome. AJR Am J Roentgenol. 2004;183:12615. https://doi.org/10.2214/ajr.183.5.1831261.

7. Lelubre C, Anselin S, Zouaoui Boudjeltia K, Biston P, Piagnerelli M. Interpretation of $\mathrm{C}$-reactive protein concentrations in critically ill patients. Biomed Res Int. 2013;2013:1-11. https://doi.org/10.1155/2013/124021.

8. Goh SL, de Silva RP, Dhital K, Gett RM. Is low serum albumin associated with postoperative complications in patients undergoing oesophagectomy for oesophageal malignancies? Interact Cardiovasc Thorac Surg. 2015;20:107-13. https://doi.org/10.1093/icvts/ivu324.

9. Hong W, Lin S, Zippi M, Geng W, Stock S, Zimmer V, et al. High-density lipoprotein cholesterol, blood urea nitrogen, and serum creatinine can predict severe acute pancreatitis. Biomed Res Int. 2017;2017:1648385. https://doi.org/10.1155/2017/1648385.

10. Lipinski M, Rydzewski A, Rydzewska G. Early changes in serum creatinine level and estimated glomerular filtration rate predict pancreatic necrosis and mortality in acute pancreatitis: creatinine and eGFR in acute pancreatitis. Pancreatology. 2013;13:207-11. https://doi.org/10.1016/j. pan.2013.02.002.

11. Lankisch PG, Weber-Dany $B$, Maisonneuve P, Lowenfels AB. High serum creatinine in acute pancreatitis: a marker for pancreatic necrosis? Am J Gastroenterol. 2010;105:1196-200. https://doi.org/10.1038/ajg.2009.688.

12. Muddana V, Whitcomb DC, Khalid A, Slivka A, Papachristou GI. Elevated serum creatinine as a marker of pancreatic necrosis in acute pancreatitis. Am J Gastroenterol. 2009;104:164-70. https://doi.org/10.1038/ ajg.2008.66.

13. Yang Z, Dong L, Zhang Y, Yang C, Gou S, Li Y, et al. Prediction of severe acute pancreatitis using a decision tree model based on the revised Atlanta classification of acute pancreatitis. PLoS ONE. 2015;10:e0143486. https://doi.org/10.1371/journal.pone.0143486.

14. Kaplan M, Ates I, Akpinar MY, Yuksel M, Kuzu UB, Kacar S, et al. Predictive value of $C$-reactive protein/albumin ratio in acute pancreatitis. Pancreat Dis Int. 2017:16:424-30. https://doi.org/10.1016/S1499-3872(17)60007-9.

15. Acevedo-Piedra NG, Moya-Hoyo N, Rey-Riveiro M, Gil S, Sempere L, Martínez J, et al. Validation of the determinant-based classification and revision of the Atlanta classification systems for acute pancreatitis. Clin Gastroenterol Hepatol. 2014;12:311-6. https://doi.org/10.1016/j. cgh.2013.07.042.

16. Nawaz H, Mounzer R, Yadav D, Yabes JG, Slivka A, Whitcomb DC, Papachristou GI. Revised Atlanta and determinant-based classification: application in a prospective cohort of acute pancreatitis patients. Am J Gastroenterol. 2013;108:1911-7. https://doi.org/10.1038/ajg.2013.348.

17. Shen X, Sun J, Zhang J, Ke L, Tong Z, Li G, et al. Risk factors and outcome for massive intra-abdominal bleeding among patients with infected necrotizing pancreatitis. Medicine (Baltimore). 2015;94:e1172. https://doi. org/10.1097/MD.0000000000001172.

18. Banks PA, Bollen TL, Dervenis C, Gooszen HG, Johnson CD, Sarr MG, et al. Classification of acute pancreatitis-2012: revision of the Atlanta classification and definitions by international consensus. Gut. 2013;62:102-11. https://doi.org/10.1136/gutjnl-2012-302779.

19. Banks PA, Freeman ML. Practice guidelines in acute pancreatitis Am J Gastroenterol. 2006;101:2379-400. https://doi.org/10.111 1/j.1572-0241.2006.00856.x.
20. Li S, Zhang Y, Li M, Xie C, Wu H. Serum albumin, a good indicator of persistent organ failure in acute pancreatitis. BMC Gastroenterol. 2017;17:59. https://doi.org/10.1186/s12876-017-0615-8.

21. Komolafe O, Pereira SP, Davidson BR, Gurusamy KS. Serum C-reactive protein, procalcitonin, and lactate dehydrogenase for the diagnosis of pancreatic necrosis. Cochrane Database Syst Rev. 2017;4:CD012645. https ://doi.org/10.1002/14651858.CD012645.

22. Azab B, Jaglall N, Atallah JP, Lamet A, Raja-Surya V, Farah B, et al. Neutrophil-lymphocyte ratio as a predictor of adverse outcomes of acute pancreatitis. Pancreatology. 2011;11:445-52. https://doi.org/10.1159/00033 1494.

23. Testoni PA. Acute recurrent pancreatitis: etiopathogenesis, diagnosis and treatment. World J Gastroenterol. 2014;20:16891-901. https://doi. org/10.3748/wjg.v20.i45.16891.

24. Matowicka-Karna J. Markers of inflammation, activation of blood platelets and coagulation disorders in inflammatory bowel diseases. Postepy Hig Med Dosw (Online). 2016;70:305-12. https://doi.org/10.5604/17322 693.1199305

25. Rhodes B, Fürnrohr BG, Vyse TJ. C-reactive protein in rheumatology: biology and genetics. Nat Rev Rheumatol. 2011;7:282-9. https://doi. org/10.1038/nrrheum.2011.37.

26. Lee KJ, Kim HM, Choi JS, Kim YJ, Kim YS, Cho JH. Comparison of predictive systems in severe acute pancreatitis according to the revised Atlanta classification. Pancreas. 2016;45:46-50. https://doi.org/10.1097/MPA.00000 00000000433.

27. Kim HJ, Lee HW. Important predictor of mortality in patients with end-stage liver disease. Clin Mol Hepatol. 2013;19:105-15. https://doi. org/10.3350/cmh.2013.19.2.105.

28. Stevens LA, Coresh J, Greene T, Levey AS. Assessing kidney function-measured and estimated glomerular filtration rate. N Engl J Med. 2006;354:2473-83. https://doi.org/10.1056/NEJMra054415.

29. Wilkman E, Kaukonen K-M, Pettilä V, Kuitunen A, Varpula M. Early hemodynamic variables and outcome in severe acute pancreatitis: a retrospective single-center cohort study. Pancreas. 2013;42:272-8. https://doi. org/10.1097/MPA.0b013e318264c9f7.

30. Suppiah A, Malde D, Arab T, Hamed M, Allgar V, Smith AM, Morris-Stiff $\mathrm{G}$. The prognostic value of the neutrophil-lymphocyte ratio (NLR) in acute pancreatitis: identification of an optimal NLR. J Gastrointest Surg. 2013;17:675-81. https://doi.org/10.1007/s1 1605-012-2121-1.

31. Kaplan M, Ates I, Oztas E, Yuksel M, Akpinar MY, Coskun O, Kayacetin E. A new marker to determine prognosis of acute pancreatitis: PLR and NLR combination. J Med Biochem. 2018;37:21-30. https://doi.org/10.1515/ jomb-2017-0039.

32. Hong W, Lin S, Zippi M, Geng W, Stock S, Basharat Z, et al. Serum albumin is independently associated with persistent organ failure in acute pancreatitis. Can J Gastroenterol Hepatol. 2017;2017:5297143. https://doi. org/10.1155/2017/5297143.

\section{Publisher's Note}

Springer Nature remains neutral with regard to jurisdictional claims in published maps and institutional affiliations.

Ready to submit your research? Choose BMC and benefit from:

- fast, convenient online submission

- thorough peer review by experienced researchers in your field

- rapid publication on acceptance

- support for research data, including large and complex data types

- gold Open Access which fosters wider collaboration and increased citations

- maximum visibility for your research: over $100 \mathrm{M}$ website views per year

At BMC, research is always in progress.

Learn more biomedcentral.com/submissions 